\title{
¿SE PUEDE PLEITEAR? PLATÓN, GARZÓN Y LA DEONTOLOGÍA DE LOS ABOGADOS
}

\author{
Liborio L. Hierro \\ Universidad Autónoma de Madrid
}

RESUMEN. Ernesto Garzón defiende que no cabe hablar de una moral institucional (y, por tanto, de una deontología o moral profesional) que sea distinta de la moral que rige las acciones individuales. Él sostiene, por el contrario, que si admitimos peculiaridades en una moral institucional (M) frente a la moral individual (M') es utilizando necesariamente una moral superior (M") que impone obligaciones especiales derivadas del rol o función social. Este trabajo desarrolla, brevemente, cuál sería el contenido de M", esto es: a) justificar el rol, b) justificar la organización institucional del rol y $c$ ) justificar los derechos y obligaciones excepcionales que se derivan del rol y de su organización.

Palabras clave: Ernesto Garzón Valdés, moral institucional, deontología.

ABSTRACT. Ernesto Garzón upholds that it is not possible to talk about institutional morality (and therefore about deontology or professional ethics) as any different from individual morality. He claims, on the contrary, that if we admit any peculiarities in institutional morality (M) as opposed to individual morality $\left(\mathrm{M}^{\prime}\right)$, then we are necessarily using a higher morality (M") which imposes special obligations derived from the social position or role. This article briefly develops the content of M", that is: a) role justification, b) the justification of the way the role is institutionally structured and $c$ ) the justification of the exceptional rights and duties derived from the role and the way it is structured.

Keywords: Ernesto Garzón Valdés, institutional morality, deontology. 
A firma PlatÓN, en «Las leyes», que si la justicia es una virtud, litigar debe ser una cosa buena, aunque se encuentra desacreditada por un «azote que se ha puesto por nombre el bello nombre de arte y que, en primer lugar, afirma que existe un procedimiento para pleitear... y que este arte, como tal, y el modo de argumentar que de ella nace, pueden serle dados a cualquiera que a cambio de ellos dé dinero» (Las leyes, XI, pp. 937-938), de lo que concluye que en una ciudad ideal no debe haber abogados y que si un ciudadano practica este ejercicio por afán de riquezas debe ser muerto y si sólo lo practica por amor propio, cuando lo haga por segunda vez también debe morir. Ernesto GARZÓN, por su parte, sostiene tres tesis que a fin de cuentas tienen que ver con esto. La primera dice que lo bueno ético es totalmente diferente de lo bueno técnico: «precisamente porque lo bueno ético se encuentra en un nivel diferente del de lo bueno técnico, tiene sentido preguntarse acerca de la calidad moral de las acciones técnicamente correctas y no es contradictorio afirmar que éstas pueden ser moralmente incorrectas» (GARZÓN, 1993: p. 545). La segunda dice que no es posible distinguir entre un ámbito de la moralidad privada y un ámbito de la moralidad pública que obedezcan a principios distintos, aun cuando cabe conceder que generalmente los problemas y las soluciones en el ámbito privado son más simples (ibidem, p. 550). La tercera dice que no hay una moral profesional específica (lo que habitualmente se denomina «deontología») ya que «el fundamento de estas obligaciones puede ser explicado también deontológicamente, recurriendo a los principios de la moral ordinaria» (ibidem, p. 550) ${ }^{1}$.

Parece que coincide GARZÓN con su buen amigo Aulis AARNIO quien llega, con similares argumentos, a la conclusión de que no existe tal cosa como la «deontología profesional». «Mi tesis principal — dice AARNIO— es que de facto no hay una ética profesional particular de los juristas (ni de ninguna otra profesión) más allá o por encima de los principios éticos que obligan a todo el mundo» (AARNIO, 2001: p. 2). Creo que la explicación última de esta posición se encuentra formulada, con su habitual claridad, en las siguientes palabras de Ernesto GARZÓN: «... los principios a los que se recurra para la justificación de las instituciones no pueden contradecir los que rigen para las acciones individuales de los hombres; admitir que hay una moral $\mathrm{M}$ para las instituciones, diferente de una moral M' que rige las acciones individuales, nos conduce necesariamente a tener que recurrir a una moral $\mathrm{M}$ " superior a ambas que nos permita decidir en caso de conflicto y adoptar una línea de acción justificada moralmente. Pero, en este caso no estamos ya frente a dos morales diferentes sino que tenemos un mismo sistema moral que impone ciertas obligaciones a una clase de personas y otras, a otras, en virtud de las distintas funciones sociales que desempeñan o de su posición en la sociedad...» (GARZÓN, 1993: p. 553).

Ejercer una profesión es, en efecto, asumir un cierto rol institucional y asumir el conjunto de obligaciones y derechos que se derivan de él ${ }^{2}$. Pero es necesario distinguir tres

${ }^{1}$ En la p. 548 GARZÓN discute la tesis de Benjamin FrEEDMAN según la cual — dice GARZÓN— «mientras la moral ordinaria responde a las características básicas de todo ser humano, la moral profesional deriva de las notas específicas de papeles sociales determinados y permite la realización de actos que desde el punto de vista de la moral ordinaria estarían prohibidos».

${ }^{2}$ KIPNIS, 1991: p. 574: «To become a good professional is, in part, to identify oneself as occupying a certain institutional role». 
tipos distintos en estos conjuntos de obligaciones y derechos profesionales. El primero es el de los derechos y obligaciones que no son en absoluto específicos aunque mantengan su vigor con ocasión del desempeño profesional; que un profesional no deba mentir a un cliente o no deba injuriar a un colega no tiene nada de específico puesto que tampoco se debe mentir a un familiar ni injuriar a un vecino; que deba realizar su trabajo con diligencia y honestidad es algo que moralmente se le puede exigir a cualquier tipo de profesional, a un comerciante o a cualquier trabajador, sea cual sea la peculiaridad de los roles que desempeña. El segundo es el de los derechos y obligaciones que sí pueden considerarse específicos en el sentido de que sólo corresponden al que ejerce una cierta profesión aunque se derivan, por conclusión o por determinación sin mayor problema, de normas morales de carácter general; su carácter específico estriba simplemente en que el que no ejerce esa profesión no se encuentra en la ocasión de aplicación de la norma (por ejemplo, si afirmamos que el médico tiene el deber moral de actualizar sus conocimientos médicos y el abogado sus conocimientos jurídicos) ${ }^{3}$. El tercero es el de los problemas interesantes, los problemas interesantes aparecen cuando comprobamos que, en muchos casos, el rol profesional requiere y justifica conductas que son contrarias a las normas morales generales y que ello es lo que ha generado ciertas costumbres, normas morales o normas jurídicas peculiares: cuando el profesional tiene el derecho o la obligación, en virtud de su rol, de hacer algo que en circunstancias normales está prohibido o cuando tiene prohibido hacer algo, en virtud de su rol, que en circunstancias normales está permitido o incluso es obligatorio ${ }^{4}$. Si esto es así, como parece, todo depende entonces de lo que diga M". Y ¿qué dice M" sobre el arte de pleitear?

Muy a pesar de PLATÓN, una llamativa peculiaridad de la actual configuración institucional de las profesiones jurídicas es que da lugar a roles profesionales que no sólo son diferentes sino contrapuestos. Aunque a veces se pretende diferenciar el sistema anglosajón de «common law» y el sistema continental de «civil law» por su respectivo carácter adversarial e inquisitorial (HAZARD, 1978: pp. 129 y 131-132) lo cierto es que, cada vez más, los sistemas continentales de aplicación del Derecho se ajustan también al diseño «adversarial» que aquí solemos denominar «contencioso» porque tiene la estructura de una contienda entre partes (MARRADI, 1983: p. 1515) y, aun cuando el juez asuma ciertas funciones inquisitoriales en la instrucción penal, ello apenas desdibuja esa estructura contenciosa y los roles distintos y contrapuestos que ella genera. Por lo que se refiere a los abogados es difícil sustraerse a la convicción dominante de que la relación del abogado con el cliente consiste, en cualquier orden de actividad, en optimizar los intereses del cliente dentro del marco de posibilidades legales. La contraposición entre el abogado-servidor-del-cliente y el abogado-colaborador-de-la administración-de-justicia es, muy plausiblemente, un dilema mal planteado pues en un sistema contencioso el abogado, ya sea en la actividad preventiva ya sea en la actividad conflictual, asume el rol de servir al cliente y es asumiendo ese rol como realiza su aportación específica a la administración de la justicia. Como dijo Lord Brougham, cuando tuvo

\footnotetext{
3 Véase en este sentido GARZÓN, 1993: p. 553: «...de la misma manera que puede decirse que los padres tienen obligaciones diferentes de las de los hijos o los solteros de los casados, sin que por ello hablemos de sistemas morales diferentes».

${ }^{4}$ Como indica Daryl KoEHn, es esta peculiaridad lo que llama la atención de los filósofos: «that professionals understand themselves as ruled by ethical norms or standards which permit, and maybe even oblige professionals, to performations not permitted by the "ordinary" norms applicable to the rest of us» (KOEHN, 1994: p. 3).
} 
que justificarse por asumir la defensa de Carolina de Brunswick contra el rey Jorge IV, en cuanto abogado la protección de su cliente era: «el primer y único deber; y que al cumplir este deber... [el abogado] debe actuar sin preocuparse de las consecuencias, aunque sea su desgraciado destino meter a su patria en la confusión».

Para dormir tranquilo tras haber metido a la patria en la confusión, o simplemente tras haber conseguido que un deudor no pague su deuda, hace falta - idesde luego! - tener un buen pretexto moral. La moral ordinaria (la M' de GARZÓN) parece ofrecer pocos pretextos para acciones de este tipo; más bien parece poner seriamente en cuestión la justificación moral de semejante actividad profesional y concede así la razón a PLATÓN. La moral especial (o institucional, la M de GARZÓN) parece ofrecer demasiados pretextos; terminaría por justificar cualquier tipo de acción requerida por razones sedicentemente técnicas en los contextos difíciles (violencias, torturas, tráficos ilícitos, contrabandos, secuestros, informaciones falsas, rebeliones armadas,...). Obviamente sólo nos cabe recurrir a M". Ernesto GARZÓN la configura como una meta norma moral que resuelve los conflictos entre M' y M. Me parece que M" tiene que prevenir esos conflictos y, para hacerlo, requiere que las obligaciones y derechos profesionales especiales que se trata de justificar satisfagan tres condiciones:

1) Que la función profesional del que se derivan esté justificada (esta primera condición podría denominarse «de la finalidad»),

2) Que la conducta excepcionalmente prohibida, obligatoria o permitida, sea una conducta instrumentalmente necesaria para cumplir la función profesional (condición «de la necesidad»), lo que se desdobla en

2.1) que sea instrumentalmente adecuada para cumplir la función (condición «de la adecuación»),

2.2) que no exista una conducta alternativa y conforme con las normas morales generales que satisfaga en los mismos términos el cumplimiento de la función (condición «de la ausencia de alternativa»),

3) Que la conducta excepcionalmente prohibida, obligatoria o permitida no implique la violación de una regla o principio de un mayor peso moral que los que son servidos por la función profesional.

La deontología normativa requiere, por tanto: a) justificar el rol, b) justificar la organización del rol y c) justificar los derechos y obligaciones excepcionales que se derivan del rol y de su organización. Este sería el contenido de M". Si ello da lugar a algo que se puede llamar «moral especial» o se trata sólo de una adecuada prolongación de la «moral ordinaria» me parece secundario. Por cierto, y para terminar por donde empecé: no creo que pleitear sea una actividad profesional moralmente supererogatoria, «cuya realización permite calificar al actor de santo o de héroe» (GARZÓN, 1993: p. 552, comentando a URMSON), pero tampoco creo — iclaro! — que esté moralmente prohibida.

\section{BIBLIOGRAFÍA CITADA}

AArnio, A., 2001: «Lawyers Professional Ethics. Do They Exist?», Ratio Iuris, vol. 14, pp. 1-9 Bobbio, N., y Matteucci, N., 1983: Diccionario de Política, Madrid: Siglo XXI (Original: Dizionario di Política, Turín: Unione Tipografico-Editrice Torinese, 1976). 
Garzón, E., 1993: Derecho, Etica y Política, Madrid: Centro de Estudios Constitucionales (se cita en particular el artículo «Moral y política», original de 1984 e incluido allí en pp. 541560).

HaZARD, G. C., 1978: Ethics in the Practice of Law, New Haven: Yale University Press.

KIPNIS, K., 1991: «Ethics and the Professional Responsability of Lawyers», Journal of Business Ethics, n. ${ }^{\circ} 10$, pp. $569-576$

KoEHn, D., 1994: The Ground of Professional Ethics, London: Routledge.

Marradi, A., 1983: Sistema judicial, en BobBio-MatteucCi, 1983: pp. 1514-1522

Platón, Las leyes; citada por la edición de J. M. PABÓn y M. Fernández Galiano, Madrid: Centro de Estudios Constitucionales, 1984. 